\title{
AN EXISTENCE THEOREM FOR A DIFFERENTIAL EQUATION OF THE SECOND ORDER, WITH AN APPLICATION TO THE CALCULUS OF VARIATIONS*
}

\author{
BY \\ GILBERT AMES BLISS
}

PiCard has shown $\uparrow$ that the equation

$$
\eta^{\prime \prime}=f\left(\xi, \eta, \eta^{\prime}\right) \ddagger
$$

has a solution $\eta=\eta(\xi)$ joining two given points $p$ and $q$, provided that $f$ satisfies certain continuity restrictions and that $q$ lies in a properly chosen region in the vicinity of the point $p$. The constants of integration are then the coördi-. nates of $p$ and $q$. In the following pages it is proposed to discuss the character of the solution as a function of these coördinates, and to extend PICARD's theorem, to the equation

$$
\frac{1}{\rho}=G\left(x, y, x^{\prime}, y^{\prime}\right),
$$

where the solution is sought in the form

$$
x=x(t), \quad y=y(t),
$$

and where

$$
\frac{1}{\rho}=\frac{x^{\prime} y^{\prime \prime}-x^{\prime \prime} y^{\prime}}{\left(x^{\prime 2}+y^{\prime 2}\right)^{?}} \text {. }
$$

In $\S 3$ an application is made to the problem of minimizing the integral

$$
I=\int_{t_{0}}^{t_{1}} F\left(x, y, x^{\prime}, y^{\prime}\right) d t
$$

taken along a curve in the form (3). It is shown that a finite closed region $S$ of the $(x, y)$-plane and a constant $\delta>0$ can be found, such that any two points

* Presented to the Society December 31, 1903. Received for publication October 29, 1903.

$\dagger$ Traité d'Analyse, vol. 3, pp. 94-100.

$\ddagger$ Primes indicate differentiation with respect to the variables $\xi, t, \tau, s$, which in the following pages do not occur in the same functions. 
of $S$ whose distance is less than $\delta$ can always be joined by a continuous extremal of the problem with a continuously turning tangent, and this extremal minimizes the integral $I$.

In his lectures* on the calculus of variations WeIERSTRAss used the first part of this result for the purpose of constructing a field in the neighborhood of a given point. The theorem also plays an essential role in the recent existence proofs of Hilbert $\dagger$ and Noble $\ddagger$ in the calculus of variations. So far as is known to the writer, however, no proofs have been given. In the proof of $\S 3$ HilberT's notion of the invariant integral has been extended to the problem of the calculus of variations for curves in parametric representation.

\section{$\S 1$. The character of Picard's solution.}

Suppose that the function $f$ in equation $(1)$ is continuous with continuous first partial derivatives $f_{\eta}, f_{\eta^{\prime}} \S$ for all values of $\xi, \eta, \eta^{\prime}$ in a region

$$
R: \quad|\xi|<2 a, \quad|\eta|<2 b, \quad\left|\eta^{\prime}\right|<2 b^{\prime},
$$

and let $M, \alpha, \beta$ be positive constants greater than the absolute values of $f, f_{\eta}, f_{\eta^{\prime}}$ respectively for such arguments. If $b^{\prime}<b_{0}^{\prime}<2 b^{\prime}$, and a region $R_{0}$ is defined entirely within $R$ by the inequalities

$$
R_{0}: \quad|\xi| \leqq a, \quad|\eta| \leqq b, \quad\left|\eta^{\prime}\right| \leqq b_{0}^{\prime},
$$

then a function

$$
\eta=\phi\left(\xi, \xi_{0}, \eta_{0}, \eta_{0}^{\prime}\right),
$$

and a positive constant $l \|$ exist with the following properties :

1) $\phi, \phi^{\prime}, \phi^{\prime \prime}$ are single-valued, continuous, and have continuous first partial derivatives with respect to $\xi_{0}, \eta_{0}, \eta_{0}^{\prime}$ for all points $\left(\xi_{0}, \eta_{0}, \eta_{0}^{\prime}\right)$ in $R_{0}$, and for $\xi \xi-\xi_{0} \mid<l$;

2) $\eta=\phi$ is a solution of the equation (1), such that $\eta, \eta^{\prime}$ have the initial values $\eta_{0}, \eta_{0}^{\prime}$ for $\xi=\xi_{0}$, and $\left(\xi, \eta, \eta^{\prime}\right)$ lies in $R$;

3) $\eta$ is the only continuous function of $\xi$ with continuous derivative on the interval $\left|\xi-\xi_{0}\right|<l$, which has the properties described in 2). $\uparrow$

* Summer, 1879.

+ Hilbert, Lectures on the calculus of variations, 1900. HiLbert extends the definition of the definite integral of the calculus of variations, making it apply to a class of functions without derivatives. The identity of this definition with one given previously by WeIkrstrass has been proved by OsGood by means of the theorem which is the subject of this paper. See the Transactions of the American Mathematical Society, vol. 2 (1901), p. 294, footnote.

$\ddagger$ NoBLE, Eine neue Methode in der Variationsrechnung, Dissertation, Göttingen, 1901.

\& Literal subscripts denote partial differentiation.

$\| l$ is the smallest of the quantities $a, b / M, 2 b^{\prime}-b_{0}^{\prime} / M$.

TF For the existence of the function (5), see, e. g., PICARD, Traité d'Analyse, vol. 2, chap. 11 ; for the uniqueness, and existence of derivatives with respect to the constants, see especially Peano, Atti di Torino, vol. 33 (1897), p. 9. Other references are given by Painlevé, 
Consider now another point $\left(\xi_{1}, \eta_{1}\right)$ besides $\left(\xi_{0}, \eta_{0}\right)$. If $\eta_{0}^{\prime}$ can be so determined that

$$
\eta_{1}=\phi\left(\xi_{1}, \xi_{0}, \eta_{0}, \eta_{0}^{\prime}\right),
$$

then the corresponding solution (5) will pass through both points. If furthermore $\partial \phi\left(\xi_{1}, \xi_{0}, \eta_{0}, \eta_{0}^{\prime}\right) / \partial \eta_{0}^{\prime} \neq 0$, then equation (6) can be solved for $\eta_{0}^{\prime}$ as a function of $\xi_{v}, \eta_{0}, \xi_{1}, \eta_{1}$, and solutions of (1) will be determined which pass through any two points in the neighborhoods of the two originally considered.

From the results of PICARD * it follows that when $\left(\xi_{1}, \eta_{1}\right)$ is properly chosen, there is one and only one solution $\eta_{0}^{\prime}$ of (6). The region about $\left(\xi_{0}, \eta_{0}\right)$

$$
R_{1}: \quad\left|\xi-\xi_{0}\right|<a, \quad\left|\eta-\eta_{0}\right|<b, \quad\left|\eta^{\prime}\right|<b_{0}^{\prime},
$$

is entirely within $R$, and consequently the assumptions for $f$ still hold true. PiCard has shown that if a positive constant $\delta$ satisfies the inequalities $\dagger$

$$
\left(\Delta_{1}\right) \quad \delta<l, \quad M_{\overline{8}}^{\delta^{2}}+\delta b^{\prime}<b, \quad M_{\overline{2}}^{\delta}+b^{\prime}<b_{0}^{\prime}, \quad \alpha \frac{\delta^{2}}{8}+\beta \frac{\delta}{2}<1,
$$

and if furthermore $\left(\xi_{1}, \eta_{1}\right)$ lies in the $\mathrm{V}$-shaped region about $\left(\xi_{0}, \eta_{0}\right)$,

$$
0<\xi_{1}-\xi_{0} \leqq \delta, \quad\left|\eta_{1}-\eta_{0}\right| \leqq\left(\xi_{1}-\xi_{0}\right) b^{\prime},
$$

then there exists a continuous solution of (1) with a continuous derivative on the interval $\xi_{0} \leqq \xi \leqq \xi_{1}$, which joins $\left(\xi_{0}, \eta_{0}\right)$ with $\left(\xi_{1}, \eta_{1}\right)$. This solution lies entirely within $R_{1}$, and is the only one with the given properties even in $R$. The initial value $\eta_{0}^{\prime}$ so defined satisfies the inequalities (4) and is the desired solution of equation (6).

Furthermore, $u=\partial \phi\left(\xi, \xi_{0}, \eta_{0}, \eta_{0}^{\prime}\right) / \partial \eta_{0}^{\prime}$ cannot vanish for such a value of $\eta_{0}^{\prime}$ when $\xi=\xi_{1}$. It is, namely, a solution of the equation

$$
u^{\prime \prime}=u f_{\eta}+u^{\prime} f_{\eta}^{\prime},
$$

which is not identically zero because at $\xi=\xi_{0}$ the initial values of $\eta, \eta^{\prime}$ are $\eta_{0}, \eta_{0}^{\prime}$, and consequently

$$
0=\frac{\partial \phi}{\partial \eta_{0}^{\prime}}, \quad 1=\frac{\partial \phi^{\prime}}{\partial \eta_{0}^{\prime}} .
$$

But according to PICARD $\ddagger$ no solution of (8) except $u \equiv 0$ vanishes twice on the interval $\xi_{0} \leqq \xi \leqq \xi_{1}$.

Enoyclopädie der Mathematischen Wissenschaften, II A 4a, pp. 195, 200. The theorem is true if $R_{0}$ is any region entirely interior to $R$, but the value of $l$ must be chosen differently.

* Traité d'Analyse, vol. 3, pp. 94-100.

† The form of statement here given differs slightly from PICARD's.

$\ddagger$ Loc. cit. p. 100. It is important to notice that the PICARD coefficients $a_{i k}, b_{i k}$ are continuous, and that the interval on which the existence proof can be made for his equations $(E)$, is the same as the interval for the original equations. 
It follows then from the foregoing and from the theorems on implicit functions * that (6) can be solved for $\eta_{0}^{\prime}$, and that the resulting function of $\xi_{0}, \eta_{0}, \xi_{1}, \eta_{1}$ is single-valued, continuous, and has continuous first partial derivatives for all values of $\xi_{0}, \eta_{0}$ which satisfy (4), and of $\xi_{1}, \eta_{1}$ which satisfy (7). After substitution of this function in (5), the latter takes the form

$$
\eta=\psi\left(\xi, \xi_{0}, \eta_{0}, \xi_{1}, \eta_{1}\right),
$$

where $\psi$ has the following properties :

1) $\psi, \psi^{\prime}, \psi^{\prime \prime}$ are single-valued and continuous, and have continuous first partial derivatives with respect to $\xi_{0}, \eta_{0}, \xi_{1}, \eta$, for all values of the arguments satisfying the conditions,

$$
\begin{gathered}
\left|\xi_{0}\right| \leqq a, \quad\left|\eta_{0}\right| \leqq b, \\
0<\xi_{1}-\xi_{0} \leqq \delta, \quad\left|\eta_{1}-\eta_{0}\right| \leqq\left(\xi_{1}-\xi_{0}\right) b^{\prime}, \\
\xi_{0} \leqq \xi \leqq \xi_{1} ;
\end{gathered}
$$

2) $\eta=\psi$ solves equation (1), has the initial and end-values $\eta_{0}, \eta_{1}$ at $\xi_{0}$ and $\xi_{1}$, and the points $\left(\xi, \eta, \eta^{\prime}\right)$ defined are all in $R$;

3) $\eta$ is the only continuous function of $\xi$ with a continuous derivative on the interval $\xi_{0} \leqq \xi \leqq \xi_{1}$, which has the properties described in 2).

\section{§2. Extension of the preceding results.}

In order to extend the results of $\S 1$ to the equation (2), suppose

1) $G$ is continuous with continuous first partial derivatives when $(x, y)$ lies in a certain finite closed region $S_{1}$ of the $(x, y)$-plane, and $x^{\prime}, y^{\prime}$ are any values not both zero;

$$
G\left(x, y, \kappa x^{\prime}, \kappa y^{\prime}\right)=G\left(x, y, x^{\prime}, y^{\prime}\right)
$$$$
(\kappa>0), \dagger
$$

for all points $\left(x, y, x^{\prime}, y^{\prime}\right)$ described in 1$)$.

If $S$ is a closed region entirely interior to $S_{1}$, a positive quantity $\delta$ can be found such that any two points $P\left(x_{0}, y_{0}\right)$ and $Q\left(x_{1}, y_{1}\right)$ of $S$, whose distance $P Q$ is less than $\delta$, can be joined by a continuous solution of (2) with a continuously turning tangent. To prove this, the $(x, y)$-plane can be transformed into a $(\xi, \eta)$-plane by translation and rotation, so that $P$ goes into the new origin and the line $P Q$ into the new $\xi$-axis. Equation (2) becomes

$$
\frac{1}{\rho}=\Gamma\left(\xi, \eta, \xi^{\prime}, \eta^{\prime}\right),
$$

* See, for example, Jordan, Ciurs d'Analyse, 2d ed., vol. 1, p. 80.

$\dagger$ This is the necessary and sufficient condition that $G$, like $\rho$, should be independent of the parametric representation. For a similar case, see KNHSER, Lehrbuch der Variationsrechnung, p. 6. 
where $\Gamma$ has the properties 1 ) and 2) with respect to a region $\Sigma_{1}$, the transform of $S_{1}$. On account of 2 ), $\xi$ can be choosen as the independent variable, so that (10) takes the form

$$
\eta^{\prime \prime}=\left(1+{\eta^{\prime}}^{2}\right)^{\xi} \Gamma\left(\xi, \eta, 1, \eta^{\prime}\right)=f\left(\xi, \eta, \eta^{\prime}\right) .
$$

Since $S$ is entirely interior to $S_{1}$, a positive constant $\epsilon$ exists such that a circle of radius $\epsilon$ about any point of $S$ lies also entirely within $S_{1}$. A region $R$ for the equation (11) is then defined by selecting $b^{\prime}$ arbitrarily and taking

$$
2 a=2 b=\frac{\epsilon}{\sqrt{2}} .
$$

The values of the constants $M, \alpha, \beta$ are independent of the choice of $P$ and $Q$ in $S$, and can be determined as follows. The absolute values of $G$ and its derivatives have a maximum $N$ when $(x, y)$ lies in $S_{1}$ and ${x^{\prime 2}}^{2}+y^{\prime 2}=1$. The corresponding maxima for $\Gamma, \Gamma_{\eta}, \Gamma_{\eta^{\prime}}$ are easily seen to be not greater than $N, 2 N, 2 N$ respectively. On account of 2 ) the absolute values of $\Gamma, \Gamma_{\eta}$ do not exceed these constants when $\xi^{\prime}, \eta^{\prime}$ take any values whatsoever which are not both zero. But

$$
\Gamma_{\eta^{\prime}}\left(\xi, \eta, \kappa \xi^{\prime}, \kappa \eta^{\prime}\right)=\frac{1}{\kappa} \Gamma_{\eta^{\prime}}\left(\xi, \eta, \xi^{\prime}, \eta^{\prime}\right)
$$

and therefore

$$
\left|\Gamma_{\eta^{\prime}}\left(\xi, \eta, \xi^{\prime}, \eta^{\prime}\right)\right|<\frac{2 N}{\sqrt{\xi^{\prime 2}+\eta^{\prime 2}}}
$$

whatever $\left(\xi^{\prime}, \eta^{\prime}\right) \neq(0,0)$ may be. The values of $M, \alpha, \beta$ are obtained from the following inequalities, derived with the help of (13):

$$
\begin{aligned}
& |f|<\left(1+4{b^{\prime}}^{2}\right)^{\frac{3}{3}} N, \\
& \left|f_{\eta}\right|=\left|\left(1+{\eta^{\prime}}^{2}\right)^{\frac{3}{3}} \Gamma_{\eta}\right|<\left(1+4{b^{\prime}}^{2}\right)^{\frac{3}{3}} \cdot 2 N, \\
& \left|f_{\eta^{\prime}}\right|=\left|\frac{3}{2}\left(1+{\eta^{\prime}}^{2}\right)^{\frac{1}{2}} \Gamma+\left(1+{\eta^{\prime}}^{2}\right)^{\frac{3}{3}} \Gamma_{\eta^{\prime}}\right|<\frac{3}{2}\left(1+4{b^{\prime 2}}^{\frac{3}{2}} N+\left(1+4{b^{\prime}}^{2}\right) 2 N . .\right.
\end{aligned}
$$

The following notations will be used;

$s=$ the length of arc measured from $P$ along a solution (3) passing through $P$;

$\tau=$ the angle between the positive $x$-axis and the direction of the tangent for increasing $s$;

$r=$ the length of the radius vector from $P$ to the point of the solution corresponding to $s$;

$\vartheta=$ the angle between the positive $x$-axis and the radius vector.

Suppose now $\delta>0$ determined as in $\S 1$ for the equation (11), and that the distance from $P$ to $Q$ is less than $\delta$. Then it follows that there exists one and 
only one continuous solution of the equation (2) with a continuously turning tangent, joining $P$ and $Q$ and satisfying the inequalities

$$
\left|r \sin \left(\vartheta-\vartheta_{1}\right)\right|<2 b, \quad\left|\tan \left(\tau-\vartheta_{1}\right)\right|<2 b^{\prime},
$$

where $\vartheta_{1}$ is the value of $\vartheta$ at $Q$. These inequalities, which are derived from the ones defining $R$, are equivalent to

$$
\left|r \sin \left(\vartheta-\vartheta_{1}\right)\right|<2 b, \quad\left|\sin \left(\tau-\vartheta_{1}\right)\right|<\frac{2 b^{\prime}}{\sqrt{1+4 b^{\prime 2}}} .
$$

The constant $\delta$ can be further restricted, however, so that every continuous solution of (2) with a continuously turning tangent, which joins $P$ and $Q$ and lies entirely within a circle of radius $\delta$ about $P$, must satisfy the inequalities (14). Consequently there can be but one such solution in the whole circle. The former of the inequalities (14) is easily seen to be true, for from $\left(\Delta_{1}\right)$ and (12)

$$
\left|r \sin \left(\vartheta-\vartheta_{1}\right)\right|<\delta<a=b .
$$

In order to make the second inequality hold true, the equations of the curve (3) can be written in the form*

$$
x-x_{0}=\int_{0}^{s} \cos \tau^{\prime} d s^{\prime}, \quad y-y_{0}=\int_{0}^{s} \sin \tau^{\prime} d s^{\prime},
$$

the primes distinguishing the variables of the integrand. Then

$$
\frac{d \tau}{d s}=\frac{1}{\rho}=G\left(x, y, \frac{d x}{d s}, \frac{d y}{d s}\right),
$$

and the square of the radius vector has the second derivative

$$
\begin{aligned}
\frac{d^{2} r^{2}}{d s^{2}} & =2\left\{1+\left(x-x_{0}\right) \frac{d^{2} x}{d s^{2}}+\left(y-y_{0}\right) \frac{d^{2} y}{d s^{2}}\right\} \\
& =2\left\{1-G\left(\sin \tau \int_{0}^{s} \cos \tau^{\prime} d s^{\prime}-\cos \tau \int_{0}^{s} \sin \tau^{\prime} d s^{\prime}\right)\right\} \\
& =2\left\{1-G \int_{0}^{s} \sin \left(\tau-\tau^{\prime}\right) d s^{\prime}\right\} \\
& \geqq 2\{1-N s\} .
\end{aligned}
$$

Since $d r^{2} / d s$ vanishes for $s=0$, the last inequality shows that $d r^{2} / d s$ and therefore $r$ constantly increase with $s$, when $s$ remains on the interval

$$
0 \leqq s \leqq \frac{1}{N}
$$

\footnotetext{
* Encyclopädie der Mathematischen Wissenschaften, III D 1, 2, p. 34.
} 
After two integrations from 0 to $s$ and a root extraction, it follows that

$$
r \geqq s \sqrt{1-\frac{1}{3} N s} \geqq s \sqrt{\frac{2}{3}} .
$$

Consequently $r$ attains the value $\sqrt{\frac{2}{3}} / N$ while $s$ is still on the interval (17). If therefore

$$
\delta<\frac{1}{N} \sqrt{\frac{2}{3}}
$$

and the arc $P Q$ is entirely within the circle of radius $\delta$ about $P$, then $s$ cannot exceed $1 / N$ and the inequality (18) holds true.

From (16) it follows that

$$
\left|\tau-\tau^{\prime}\right|=\left|\int_{s^{\prime}}^{s} G d s\right| \leqq N\left|s-s^{\prime}\right|,
$$

where $\tau^{\prime}$ is the value of $\tau$ at any point $\left(s=s^{\prime}\right)$ of the arc $P Q$. Further, from (15),

$$
\sin \left(\tau-\vartheta_{1}\right)=\sin \tau \cdot \frac{x_{1}-x_{0}}{r_{1}}-\cos \tau \cdot \frac{y_{1}-y_{0}}{r_{1}}=\frac{1}{r_{1}} \int_{0}^{s_{1}} \sin \left(\tau-\tau^{\prime}\right) d s^{\prime},
$$

where $r_{1}, s_{1}$ are the values of $r, s$ at $Q$. Consequently, from (19) and (18),

$$
\left|\sin \left(\tau-\vartheta_{1}\right)\right| \leqq \frac{1}{r_{1}} \int_{0}^{s_{1}}\left|\tau-\tau^{\prime}\right| d s^{\prime} \leqq N \frac{s_{1}^{2}}{2 r_{1}} \leqq \frac{3}{4} N r_{1},
$$

and the second of the inequalities (14) will be satisfied if

$$
\frac{3}{4} N \delta<\frac{2 b^{\prime}}{\sqrt{1+4 b^{\prime 2}}} .
$$

It has been proved therefore that there is one and but one continuous solution of (2) with a continuously turning tangent, which joins $P$ and $Q$ and lies entirely within a circle of radius $\delta$ about $P$, provided that $\delta$ satisfies $\left(\Delta_{1}\right),\left(\Delta_{2}\right)$ and $\left(\Delta_{3}\right)$. If the length $s$ along the arc $P Q$ is taken as parameter, the equations of this solution can easily be found. The relations between the $(x, y)$ - and $(\xi, \eta)$-coördinates are

$$
x=x_{0}+\xi \cos \vartheta_{1}-\eta \sin \vartheta_{1}, \quad y=y_{0}+\xi \sin \vartheta_{1}+\eta \cos \vartheta_{1},
$$

and the relation between $\xi$ and $s$ is

$$
\int_{\xi_{0}}^{\xi} \sqrt{1+\eta^{\prime 2}} d \xi=s
$$

On account of the theorems on implicit functions and the properties of (9), the equation (21) has a solution 


$$
\xi=\xi\left(s, \xi_{0}, \eta_{0}, \xi_{1}, \eta_{1}\right),
$$

where the function $\xi$, together with its derivatives $\xi^{\prime}, \xi^{\prime \prime}$, is continuous and has continuous first partial derivatives with respect to $\xi_{0}, \eta_{0}, \xi_{1}, \eta_{1}$ for all points $\left(\xi_{0}, \eta_{0}\right)$ and $\left(\xi_{1}, \eta_{1}\right)$ within small circles about $(0,0)$ and $\left(r_{1}, 0\right)$ respectively, and for

$$
0 \leqq s \leqq s_{1}=\int_{\xi_{0}}^{\xi_{1}} \sqrt{1+\eta^{\prime 2}} d \xi
$$

This function $\xi$ can now be substituted in (9), and then both (22) and (9) in $(20)$. The resulting equations give all the solutions of (2) joining points in the neighborhood of $P$ and $Q$. If $\xi_{0}, \eta_{0}, \xi_{1}, \eta_{1}$ are replaced by their values in $(x, y)$-coördinates, it follows that there exist two functions

$$
x=g\left(s, x_{0}, y_{0}, x_{1}, y_{1}\right), \quad y=h\left(s, x_{0}, y_{0}, x_{1}, y_{1}\right)
$$

with the properties:

1) $g^{\prime}, g^{\prime \prime}, h^{\prime}, h^{\prime \prime}$ exist and with $g, h$ are single-valued and continuous, and have continuous first partial derivatives with respect to $x_{0}, y_{0}, x_{1}, y_{1}$ for all values of the five arguments such that

a) $\left(x_{0}, y_{0}\right)$ is in $S$, and $\sqrt{\left(x_{1}-x_{0}\right)^{2}+\left(y_{1}-y_{0}\right)^{2}}<\delta$,

b) $0 \leqq s \leqq s_{1}$; *

2) the curve defined by (24) lies entirely in a circle of radius $\delta$ about $\left(x_{0}, y_{0}\right)$, solves equation (2), and passes through $\left(x_{0}, y_{0}\right),\left(x_{1}, y_{1}\right)$, for $s=0$ and $s=s_{1}$ respectively:

3) this is the only continuous curve with continuously turning tangent which has the properties described in 2).

\section{§3. Application to the calculus of variations.}

In the integral $I$ suppose that the function $F$ is continuous with continuous first, second and third partial derivatives, and that

$$
\begin{gathered}
F\left(x, y, \kappa x^{\prime}, \kappa y^{\prime}\right)=\kappa F\left(x, y, x^{\prime}, y^{\prime}\right) \\
F_{1}=\frac{1}{y^{\prime 2}} F_{x^{\prime} x^{\prime}}=-\frac{1}{x^{\prime} y^{\prime}} F_{x^{\prime} y^{\prime}}=\frac{1}{x^{\prime 2}} F_{y^{\prime} y^{\prime}}>0, \ddagger
\end{gathered}
$$

\footnotetext{
* From equation (23) it follows that $s_{1}$ is a continuous function of $x_{0}, y_{0}, x_{1}, y_{1}$ with continuous first partial derivatives.

† This is the condition for the integral $I$ to be invariant under change of parametric representation. See KNESER, loc. cit.

$\ddagger$ For the possibility of defining $F_{1}$ in this way see KNeser, loc. oit. p. 52. Problems for which $F_{1}>0$ have been named regular by Hilbert ; Mathematical Problems, Bulletin of the American Mathematical Society, ser. 2, vol. 8 (1902), p. 469.
} 
for all points $(x, y)$ in a finite closed region $\dot{S}_{1}$ of the $(x, y)$-plane, and for any values of $x^{\prime}, y^{\prime}$ which are not both zero. EuLer's equation for $I$ is

$$
F_{x y^{\prime}}-F_{x^{\prime} y}+F_{1}\left(x^{\prime} y^{\prime \prime}-x^{\prime \prime} y^{\prime}\right)=0, *
$$

or

$$
\frac{1}{\rho}=\frac{F_{x^{\prime} y}-F_{x y^{\prime}}}{\left(x^{\prime 2}+y^{\prime 2}\right)^{\frac{y}{3} F_{1}}}=G\left(x, y, x^{\prime}, y^{\prime}\right),
$$

which has the properties of equation (2) in the region $S_{1}$. Any two points $P\left(x_{0}, y_{0}\right)$ and $R\left(x_{2}, y_{2}\right)$ in a region $S$, and such that the distance $P R$ is less than $\delta$, can therefore be joined by an extremal $\mathbb{E}$, one of the set (24). Let $\mathbb{E}$ denote any other continuous curve of the form (3) joining $P$ and $R$, and consisting of a finite number of pieces, each of which has a continuously turning tangent. Then it is desired to prove that $I_{\mathbb{E}}$, the value of $I$ taken along $\mathbb{E}$ from $P$ to $R$, is smaller than the value $I_{\mathfrak{E}}$ taken along $\mathfrak{C}$.

Suppose that the curve $\mathbb{C}$ passes through $P$ for $t=t_{0}$ and through $R$ for $t=t_{2}$, and in the integral

$$
I_{\mathfrak{s}}^{*}=\int_{t_{0}}^{t_{2}}\left\{x^{\prime} F_{p}(x, y, p, q)+y^{\prime} F_{q}(x, y, p, q)\right\} d t, \dagger
$$

let $p$ and $q$ be functions of $x, y$ defined by the equations

$$
p(x, y)=g^{\prime}\left(s_{1}, x_{0}, y_{0}, x, y\right), \quad q(x, y)=h^{\prime}\left(s_{1}, x_{0}, y_{0}, x, y\right),
$$

in which $x_{0}$ and $y_{0}$ are kept constant and $x_{1}, y_{1}$ are replaced by $x, y$. Then the value $I_{\mathscr{G}}^{*}$ is independent of the curve $\mathbb{C}$. For if $U, V$ represent the coefficients of $x^{\prime}, y^{\prime}$ in the integrand respectively, the criterion for independence of path is that

$$
\frac{\partial U}{\partial y} \equiv \frac{\partial V}{\partial x}
$$

over the whole circle of radius $\delta$ about $P . \ddagger \quad$ But

$$
\begin{aligned}
& \frac{\partial U}{\partial y}=F_{p y}+F_{p p} p_{y}+F_{p q} q_{y}=F_{p y}+F_{1} q\left(q p_{y}-p q_{y}\right), \\
& \frac{\partial V}{\partial x}=F_{q x}+F_{q p} p_{x}+F_{q q} q_{x}=F_{q x}+F_{1} p\left(-q p_{x}+p q_{x}\right)
\end{aligned}
$$

* WhimRstrass, Lectures, 1879.

†This is HILBERT's invariant integral for the problem in parametric representation. Compare with OsGood, Annals of Mathematics, ser. 2, vol. 2 (1901), p. 121. The expressions $F_{p}, F_{q}$, etc., will be used to denote partial derivatives of $F$ with the arguments $p, q$ instead of $x^{\prime}, y^{\prime}$.

$\ddagger$ PICARD, Traité d'Analyse, vol. 1, p. 85 . 
on account of $(26)$, and therefore

$$
\frac{\partial V}{\partial x}-\frac{\partial U}{\partial y} \equiv F_{q x}-F_{p y}+F_{1}\left\{p\left(q_{x} p+q_{y} q\right)-q\left(p_{x} p+p_{y} q\right)\right\}
$$

The differential equations

$$
\frac{d x}{d s}=p(x, y), \quad \frac{d y}{d s}=q(x, y)
$$

are satisfied by all the solutions (24) which pass through the point $P$, so that

$$
\frac{d^{2} x}{d s^{2}}=p_{x} p+p_{y} q, \quad \frac{d^{2} y}{d s^{2}}=q_{x} p+q_{y} q .
$$

The expression (28) is the left hand side of (27) with $x$ and $y$ replaced by the functions (24) in which, furthermore, $s=s_{1}$. It vanishes identically in $x$ and $y$.

Consider the difference

On account of (25)

$$
\Delta I=I_{\mathfrak{E}}-I_{\mathfrak{E}} .
$$

and therefore

$$
F\left(x, y, x^{\prime}, y^{\prime}\right)=x^{\prime} F_{x^{\prime}}+y^{\prime} F_{y^{\prime}},
$$

$$
I_{\mathbb{E}}=\int_{s_{0}}^{s_{1}}\left\{F_{p} \cdot p+F_{q} \cdot q\right\} d s=I_{\mathbb{E}}^{*}=I_{\mathfrak{E}}^{*} .
$$

It follows then that

$$
\Delta I=I_{\varpi}-I_{\mathbb{E}}^{*}=\int_{t_{0}}^{t_{1}} E\left(x, y, p, q, x^{\prime}, y^{\prime}\right) d t,
$$

where $p, q$ and $x^{\prime}, y^{\prime}$ determine the directions of $\mathfrak{E}$ and $\mathfrak{C}$ respectively, and

$$
E=F\left(x, y, x^{\prime}, y^{\prime}\right)-x^{\prime} F_{p}-y^{\prime} F_{q} .
$$

According to Weierstrass,*

where

$$
E=(1-\cos l) F_{1}\left[x, y, \cos \left(\lambda+l_{1}\right), \sin \left(\lambda+l_{1}\right)\right],
$$

$$
\begin{gathered}
\cos \lambda=p, \quad \sin \lambda=q, \\
\cos (\lambda+l)=\frac{x^{\prime}}{\sqrt{x^{\prime 2}+y^{\prime 2}}}, \quad \sin (\lambda+l)=\frac{y^{\prime}}{\sqrt{x^{\prime 2}+y^{\prime 2}}},
\end{gathered}
$$

and $0 \leqq l_{1} \leqq l$. The function $E$ is consequently positive at all points where $l \neq 0$, and therefore $\Delta I>0$ unless $l \equiv 0$ along the curve $\mathfrak{C}$.

To prove that $l \neq 0$ unless $\mathfrak{C}$ and $\mathbb{E}$ coincide, suppose the contrary. Then

* Lectures on the ca'culus of variations, 1882. 


$$
\frac{x^{\prime}}{\sqrt{{x^{\prime 2}+y^{\prime 2}}^{2}}}=p(x, y), \quad \frac{y^{\prime}}{\sqrt{{p^{\prime 2}}^{2}+q^{\prime 2}}}=q(x, y)
$$

along the whole are $P Q$ of $\mathfrak{C}$. It follows from this equation that $\mathfrak{C}$ has a continuously turning tangent and solves equation (27). The curvature of $\mathbb{C}$ is, namely, from the last two equations,

$$
\frac{1}{\rho}=p\left(q_{x} p+q_{y} q\right)-q\left(p_{x} p+p_{y} q\right) .
$$

Therefore $\mathbb{C}$ and $\mathbb{E}$ must coincide on account of the uniqueness of the solution determined by $P$ and $Q$.

The following theorem has been proved:

The function $F$ in the integral $I$ is supposed to have the properties described at the beginning of $\S 3$, when $(x, y)$ is in a finite closed region $S_{1}$ of the $(x, y)$-plane and for all values of $x^{\prime}, y^{\prime}$ which are not both zero. $S$ is a closed region interior to $S_{1}$.

Then a positive quantity $\delta$ can be determined such that any two points $P$ and $R$ of $S$, whose distance is less than $\delta$, can be joined by one and only one continuous solution of Euler's equation which has a continuously turning tangent and lies entirely in a circle of radius $\delta$ about the point $P$. This solution gives to 1 a smaller value than any other continuous curve which 1) joins $P$ and $R, 2)$ lies in the circle, and 3) consists of a finite number of pieces, each of which has a continuously turning tangent.

If the hypothesis, $F>0$ for all values of $x, y, x^{\prime}, y^{\prime}$ such that $(x, y)$ is in $S_{1}$ and $x^{\prime}, y^{\prime}$ are not both zero, is added, then a still stronger statement can be made concerning the extremal $P Q$. Each one of the extremals (24) has a direction $\tau_{0}$ at the point $P$ and the set can therefore be represented in the form

$$
x=\bar{g}\left(s, x_{0}, y_{0}, \tau_{0}\right), \quad y=\bar{h}\left(s, x_{0}, y_{0}, \tau_{0}\right) .
$$

By reasoning similar to the preceding for equations (24), it can be shown from the results in $\S 1$ that these functions, together with $\bar{g}^{\prime}, \bar{h}^{\prime}, \bar{g}^{\prime \prime}, \bar{h}^{\prime \prime}$, are continuous and have continuous first partial derivatives with respect to $x_{0}, y_{0}, \tau_{0}$ for all values of $\left(x_{0}, y_{0}\right)$ in the region $S$, of $\tau_{0}$ such that

$$
-\pi \leqq \tau_{0} \leqq+\pi,
$$

and of $s$ corresponding to points in the circle of radius $\delta$ about $P$.

Suppose $m>0$ to be the minimum of $F$ when $(x, y)$ is in the closed region $S_{1}$ and $x^{\prime 2}+y^{\prime 2}=1$. Points on the circumference of the circle of radius $\delta$ are defined by values $s \geqq \delta$, and consequently the value of $I$ taken along an extremal from $P$ to the circumference is $\geqq m \delta$. It follows that if $i$ satisfies 


$$
0<i \leqq m \delta,
$$

then $\sigma$ can be so determined for each value of $\tau_{0}$ that

$$
\int_{0}^{\sigma} F\left(\bar{g}, \bar{h}, \bar{g}^{\prime}, \bar{h}^{\prime}\right) d s=i .
$$

The function $\sigma\left(\tau_{0}, i\right)$ so defined is single-valued, continuous, and has continuous first partial derivatives for all values of $\tau_{0}$ and of $i$ on the intervals (30) and (31) respectively. The curve

$$
x=\bar{g}\left(\sigma, x_{0}, y_{0}, \tau_{0}\right)=x\left(\tau_{0}\right), \quad y=\bar{h}\left(\sigma, x_{0}, y_{0}, \tau_{0}\right)=y\left(\tau_{0}\right)
$$

is a closed continuous curve $\mathfrak{C}_{i}{ }^{*}$ about the point $P$ and has a continuously turning tangent. Furthermore the extremal joining $P$ with any other point interior to $\mathfrak{C}_{i}$ gives to $I$ a value less than $i$. If $R$ is a point within the curve $\mathfrak{C}_{m \delta}$, then the extremal $P R$ gives $I$ a smaller value than that given by any other curve $\mathbb{E}$ whatsoever joining $P$ and $R$ in $S_{1}$. For the extremal minimizes $I$ with respect to all curves joining $P$ and $R$ and lying in the circle of radius $\delta$ about $P$. Any other curve $\mathbb{C}$ which crosses the circle must also intersect the curve $\mathfrak{C}_{m \delta}$, and at the point of intersection the value of $I$ will already be as large as $m \delta$. Since $F>0$, it follows that the value of $I$ taken along the whole arc from $P$ to $R$ must be still greater.

It remains to show that $a$ positive constant $\delta_{1}$ can be found such that a circle of radius $\delta_{1}$ about any point $P$ of the region $S$ lies within the corresponding curve $\mathfrak{S}_{m \delta}$; that is to say, the minimizing property holds uniformly over the whole region $S$. This follows because from (18) and (32) the radius vector of any point on $\mathfrak{C}_{m \delta}$ satisfies the inequalities

$$
r \geqq \sqrt{\frac{2}{3}} \sigma\left(\tau_{0}, m \delta\right) \geqq \sqrt{\frac{2}{3}} \frac{m}{M} \delta,
$$

where $M$ is the maximum of $F^{\prime}$ in the region $S_{1}$.

An important illustration of the preceding results is furnished by the geodesic coördinates upon a surface. In this case the integral is

$$
I=\int_{t_{0}}^{t_{1}} \sqrt{E\left(\frac{d u}{d t}\right)^{2}+2 F \frac{d u}{d t} \frac{d v}{d t}+G\left(\frac{d v}{d t}\right)^{2}} d t,
$$

where the surface is taken in the form

$$
x=x(u, v), \quad y=y(u, v), \quad z=z(u, v),
$$

* These curves are the so-called "transversals," KNESER, loc. cit., p. 32. 
and $E, F, G$ have the usual meaning. If in the $(u, v)$-region considered the surface has no singularities, and the functions (33) have continuous first and second partial derivatives, then the function under the integral sign satisfies all the hypotheses of $\S 3$, and also $F>0$. The set of extremals $(29)$ is the totality of geodesic lines through the point $P$; the curves $\mathfrak{C}_{i}$ are the geodesic circles about that point; and the geodesic coördinates are $\tau_{0}, i$.

The University of Chicago. 